\title{
20 Years of CORAVEL Monitoring of Radial-Velocity Standard Stars
}

S. Udry ${ }^{1}$, M. Mayor ${ }^{1}$, E. Maurice ${ }^{2}$, J. Andersen ${ }^{3}$, M. Imbert ${ }^{2}$, H. Lindgren $^{4}$, J.-C. Mermilliod ${ }^{5}$, B. Nordström ${ }^{3}$, L. Prévot ${ }^{2}$

${ }^{1}$ Observatoire de Genève, 51 ch. des Maillettes, CH-1290 Sauverny

${ }^{2}$ Observatoire de Marseille, 2 place Le Verrier, F-13248 Marseille

${ }^{3}$ Niels Bohr Institute, Juliane Maries Vej 30, DK-2100 Copenhagen OE

${ }^{4}$ Lund Observatory, Box 43,5 -22100 Lund

${ }^{5}$ Institut d'astronomie, Université de Lausanne, CH-1290

Chavannes-des-bois

\begin{abstract}
A preliminary analysis of a sample of more than hundred stars, used as radial-velocity standards and followed with the CORAVEL spectrographs over 1 to 2 decades, is presented. Stars with intrinsic variability or orbital motions are pointed out.
\end{abstract}

\section{Introduction}

During more than 20 years a group of 111 stars has been monitored with the two CORAVEL spectrometers as radial-velocity standard stars. The large number of measurements ( $N_{\text {tot }} \simeq 20350$ ) and their very long time-base (Fig. 1) provide an unequalled sample to check the long term stability of radial-velocity standards. The long term survey permits the rejection of low-amplitude and long-period binaries and of intrinsic variable stars.

One of the aims of the analysis of the CORAVEL standards is to provide a list of constant stars usable as radial-velocity standards, extending the list proposed by the IAU. In most cases, it is however strongly recommended to use the new high-precision standard list defined by combining CORAVEL (very long time base) and ELODIE (high precision) measurements (Udry, Mayor \& Queloz 1999).

The subsample of standard stars constant in radial velocity has been used to follow and correct the time-dependent drift of the CORAVEL spectrographs.

\section{Origin of the sample}

The CORAVEL standard stars have been chosen from different lists in order to cover a large range in colour, velocity and magnitude (Table 1): the bright and faint IAU standard stars (Pearce 1955; IAUb, IAUf in Table 1), a suggested extension of northern faint standards (Heard 1968; $\mathrm{H}$ in Table 1), a suggested extension of southern faint standards (Evans 1968; E in Table 1), a selected sam- 

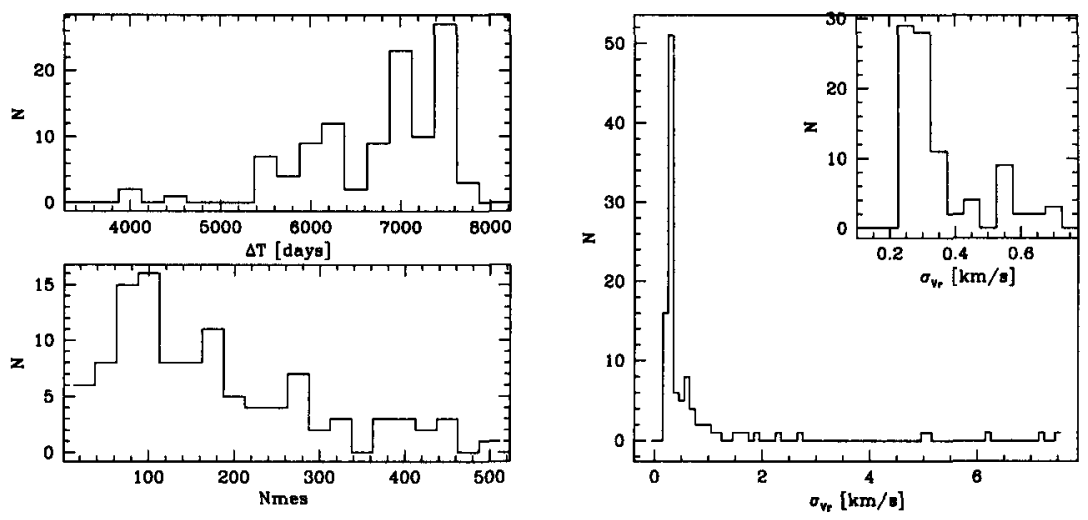

Figure 1. Left. Distributions of the number of measurements per star and of the spans in days $(\Delta T)$ between their first and last observations. Right. Histogram of the radial-velocity dispersion of the measurements. The right-hand tail includes the stars with recognized orbital motion or stars showing intrinsic variations.

ple of stars from the Wilson catalogue (1953) with high (quality a) or moderate (quality b) velocities ( $\mathrm{Wa}$ and $\mathrm{Wb}$ in Table 1 ).

From our sample, four stars chosen as standards by R.F. Griffin and also intensively followed with CORAVEL are not included here: HD 3346, HD 54716 , HD 113996 and HD 176670. They will be discussed elsewhere.

\section{Result summary}

The variability analysis of 107 among the 111 stars forming the initial CORAVEL catalogue of standards (including the IAU standards) is presented in Fig. 1 (right) and Table 1. At a precision level of $\sim 0.3 \mathrm{~km} \mathrm{~s}^{-1}$, a large number among these stars ( $\sim 70$ stars) are found to be constant (blank in the 'Rem' column of Table 1) over a very long period of time, providing thus a useful, homogeneous sample of standard stars. In particular, they extend the IAU list towards the large velocity values. In the table the radial velocities are given in the "ELODIE" system (Udry et al. 1999), rounding off the values at a $100 \mathrm{~m} \mathrm{~s}^{-1}$ precision level. They supersede the values given in Mayor \& Maurice (1985).

The radial-velocity variable stars split in different categories defined by the most probable causes of the observed velocity variations: orbital motion, intrinsic processes or unknown.

For 13 stars of the sample the velocity variations relate to orbital motions. 7 have well determined orbital parameters (SBO or SB2O in Table 1) among which three are new: HD $42397(P=5203.3 \mathrm{~d}$; the SB2 feature was already pointed out by Scarfe 1992), HD $86801(P=9972.1 \mathrm{~d}, e=0.9)$ and HD 101266 $\left(P=3805.2 \mathrm{~d}, m_{2} \sin i=0.076 \mathrm{M}_{\odot}\right)$. For the other four binaries, the orbital elements were respectively derived by Mazeh, Latham, \& Stefanik (1996) and 
Mayor et al. (1997) for HD 29587 and HD 140913 , by Latham et al. (1989) for HD 114762, and by McClure (1983) for HD 184467 . The CORAVEL orbits will be given in the main paper describing the CORAVEL standard star sample (Udry et al., in preparation). In addition to those binaries, 6 stars show a very clear drift in radial velocity over a span of more than 6000 days. Two of those are giants and thus could experience intrinsic variations (see below) but the timescale and amplitude of the variations point towards an orbital explanation for the observed drift.

Among the other radial-velocity variables, several red and yellow giants present variations with well defined periodicities. In particular 5 of them $(2$ new ones) have large radial-velocity variations and/or long periods and were thus considered first as binaries. However, the long-P radial-velocity variations of yellow giants are now associated with intrinsic phenomena (Larson, Yang, \& Walker 1999; Walker et al. 1989). The 5 candidates, indicated by SBO-IVAR? in Table 1, are the following: HD $14969\left(P=1911.6 \mathrm{~d}, K=4.56 \mathrm{~km} \mathrm{~s}^{-1}\right.$, Griffin 1980), HD $35410\left(P=1491.2 \mathrm{~d}, K=1.94 \mathrm{~km} \mathrm{~s}^{-1}\right.$, new $)$, HD 44131 $\left(P=4043.1 \mathrm{~d}, K=1.26 \mathrm{~km} \mathrm{~s}^{-1}\right.$, new $)$, HD $160952(P=181.7 \mathrm{~d}, K=2.57$ $\mathrm{km} \mathrm{s}^{-1}$, Radford \& Griffin 1976) and HD $204934(P=143.79 \mathrm{~d}, K=6.14 \mathrm{~km}$ $\mathrm{s}^{-1}$, Radford \& Griffin 1975). A group of 5 additional giant stars of spectral types between K3 and M4 (HD 108903, HD 115521, HD 123782, HD 186791 , HD 223094) show smaller amplitude variations $\left(\sigma_{V r}<0.6 \mathrm{~km} \mathrm{~s}^{-1}\right)$ with well defined time-scales of a few hundred days. Moreover, the variation phases seem to be fairly well conserved over the full time span of our observations i.e. over 10 to 20 cycles of variation. The star HD 3346 (McClure et al. 1985) presents the same characteristics. From our data, a period-amplitude-colour trend can be pointed out for varying giant stars. This trend will be further examined in the future.

Finally, associated with the very high precision of the ELODIE spectrograph, the long time-base of the CORAVEL measurements allows us to propose a list of high-precision radial-velocity standards for solar-type stars ( $\mathrm{G}$ and $\mathrm{K}$ dwarfs). This new high-precision standard sample is given in Udry et al. (1999).

Acknowledgments. It is a pleasure to thank the technical support team of the CORAVEL spectrographs and the various CORAVEL observers for their regular and diligent measures of standards. This work has benefited from the continuous support of the Fonds National Suisse de la Recherche Scientifique.

\section{References}

Evans, D.S. 1968, in Trans. IAU, XIII B, L. Perek, Dordrecht: Reidel, 170 Griffin, R.F. 1980, MNRAS, 190, 711

Heard, J.F. 1968, in Trans. IAU, XIII B, L. Perek, Dordrecht; Reidel, 169

Larson, A.M., Yang, S.L.S., \& Walker, G.A.H. 1999, these Proceedings

Latham, D., Mazeh, T., Stefanik, R., Mayor, M., \& Burki, G. 1989, Nature 339, 38

Mayor, M., \& Maurice, E. 1985, in Stellar Radial Velocities (IAU Coll. 88), A.G.D. Philip \& D.W. Latham, Schenectady, L.Davis, 299 
Mayor, M., Queloz, D., Udry, S., \& Halbwachs, J.-L. 1997, in Astronomical and Biochemical Origins and the Search for Life in the Universe (IAU Coll. 161), C. Cosmovici, S. Bowyer \& D. Werthimer, Bologna: Editrice Compositori, 313

Mazeh, T., Latham, D., \& Stefanik, R. 1996, ApJ, 466, 415

McClure R.D. 1983, PASP, 95, 201

McClure, R.D., Griffin, R.F., Fletcher, J.M., Harris, H.C., \& Mayor, M. 1985, PASP, 97, 740

Pearce, J.A. 1955, in Trans. IAU, IX B, P. Oosterhoff, Cambridge: Camb. Univ. Press, 441

Radford, G.A., \& Griffin, R.F. 1975, Observatory, 95, 187

Radford, G.A., \& Griffin, R.F. 1976, Observatory, 96, 56

Scarfe, C.D. 1992, Information Bulletin on Variable Stars, 3736

Udry, S., Mayor, M., \& Queloz, D. 1999, these Proceedings

Walker, G., Yang, S., Campbell, B., \& Irwin, A. 1989, ApJ, 343, L21

Wilson, R.E. 1953, General Catalogue of Stellar Radial Velocities, Washington: Carnegie Institution of Washington

Table 1.: Overview of the CORAVEL radial-velocity standard stars. The first 4 columns give the identification and photometric information. Columns 5 and 6 provide the CORAVEL radial velocity and velocity dispersion $\left(\mathrm{km} \mathrm{s}^{-1}\right)$ in the new "ELODIE" system (Udry et al. 1999). Column 7 provides the source-list of the standards and the last column gives information on the probable cause of variability: $S B O, S B 2 O$, Drift refer to an orbital motion, IVAR indicates a probable intrinsic variability whereas $V A R$ is used for variability whose origin is still unclear.

\begin{tabular}{lrlrrrll}
\hline HD/DM & V & Sp & B-V & \multicolumn{1}{c}{$V_{r}$} & $\sigma_{V r}$ & list & Rem \\
\hline 26 & 8.26 & G4V & 1.05 & -215.3 & 1.2 & Wb & Drift \\
693 & 4.89 & F5V & 0.49 & 15.0 & 0.3 & IAUf & \\
3712 & 2.24 & K0II-III & 1.17 & -4.3 & 0.2 & IAUb & \\
3765 & 7.36 & K2V & 0.94 & -63.3 & 0.2 & IAUf & \\
4128 & 2.04 & K0III & 1.02 & 13.1 & 0.2 & IAUb & \\
4388 & 7.34 & K3III & 1.12 & -27.5 & 0.3 & H & \\
6655 & 8.05 & F8V & 0.56 & 19.5 & 0.3 & E & \\
8779 & 6.42 & K0IV & 1.24 & -4.2 & 0.3 & IAUf & \\
9138 & 4.84 & K4III & 1.37 & 34.2 & 0.2 & IAUf & \\
12029 & 8.2 & K2III & 0.40 & 38.5 & 0.3 & H & \\
12929 & 2.01 & K2III & 1.15 & -14.6 & 0.2 & IAUb & \\
14969 & 7.07 & K3III & 1.42 & -32.9 & 2.7 & H & SBO-IVAR? \\
18884 & 2.54 & M2III & 1.63 & -26.1 & 0.3 & IAUb & \\
20902 & 1.79 & F5Ib & 0.48 & -2.0 & 0.6 & IAUb & IVAR \\
22484 & 4.29 & F9V & 0.57 & 27.9 & 0.3 & IAUf & \\
22879 & 6.68 & F9V & 0.55 & 120.2 & 0.3 & Wb & \\
\hline
\end{tabular}




\begin{tabular}{|c|c|c|c|c|c|c|c|}
\hline HD/DM & $\bar{V}$ & $\mathrm{Sp}$ & $\overline{\mathrm{B}-\mathrm{V}}$ & $\overline{V_{r}}$ & $\sigma_{V r}$ & list & Rem \\
\hline 23169 & 8.5 & G2V & 0.60 & 14.2 & 0.3 & $\overline{\mathrm{H}}$ & \\
\hline 23614 & 4.43 & M1III & 1.60 & 45.2 & 0.5 & Wa & IVAR \\
\hline 24331 & 8.61 & $\mathrm{~K} 2 \mathrm{~V}$ & 0.91 & 23.9 & 0.2 & $E$ & \\
\hline 25025 & 2.97 & M1IIIb & 1.59 & 61.1 & 0.5 & Wa & IVAR \\
\hline 25532 & 8.20 & F6IV-V & 0.66 & -111.5 & 0.4 & $\mathrm{~Wb}$ & VAR \\
\hline 26162 & 5.51 & K2III & 1.08 & 24.8 & 0.3 & IAUf & \\
\hline 29139 & 0.87 & K5III & 1.54 & 54.2 & 0.2 & IAUb & \\
\hline 29587 & 7.29 & G2V & 0.63 & 112.4 & 0.5 & IAUf & $\mathrm{SBO}$ \\
\hline 32963 & 7.60 & G5IV & 0.66 & -62.4 & 0.3 & $\mathrm{H}$ & \\
\hline 35410 & 5.07 & K0III & 0.96 & 20.7 & 0.7 & IAUf & New SBO-IVAR? \\
\hline 36079 & 2.81 & G5II & 0.81 & -14.2 & 0.3 & IAUb & \\
\hline 36673 & 2.58 & F0Ib & 0.21 & 25.2 & 1.0 & IAUb & IVAR \\
\hline 37160 & 4.09 & G8III-IV & 0.95 & 99.0 & 0.3 & $\mathrm{Wa}$ & \\
\hline 39194 & 8.09 & $\mathrm{KOV}$ & 0.76 & 13.9 & 0.3 & $\mathrm{E}$ & \\
\hline 39364 & 3.76 & G8III-IV & 0.98 & 100.2 & 0.3 & Wa & \\
\hline 42397 & 7.7 & G0IV & 0.90 & 38.3 & 9.0 & $\mathrm{H}$ & New SB2O \\
\hline 44131 & 4.91 & M1III & 1.61 & 48.7 & 0.9 & IAUf & New SBO-IVAR? \\
\hline$-43: 2527$ & 8.61 & K1III & 1.15 & 19.7 & 0.9 & $\mathrm{E}$ & VAR \\
\hline 48381 & 8.49 & K5 & 1.05 & 40.5 & 0.2 & $\mathrm{E}$ & \\
\hline 50778 & 4.08 & K4III & 1.42 & 96.2 & 0.4 & $\mathrm{Wa}$ & \\
\hline 51250 & 5.00 & B9.5V & 1.18 & 18.1 & 0.3 & IAUf & \\
\hline 54810 & 4.91 & K0III & 1.02 & 77.5 & 0.3 & $\mathrm{Wa}$ & \\
\hline 62509 & 1.16 & K0III & 0.99 & 3.2 & 0.3 & IAUb & \\
\hline 65583 & 6.97 & G8V & 0.72 & 14.7 & 0.3 & IAUf & \\
\hline 65934 & 7.70 & G8III & 0.93 & 35.8 & 0.3 & $\mathrm{H}$ & \\
\hline 66141 & 4.39 & K2III & 1.25 & 71.6 & 0.3 & IAUf & \\
\hline 75935 & 8.46 & G8V & 0.77 & -18.1 & 0.3 & $\mathrm{H}$ & \\
\hline 76932 & 5.80 & F7-F8IV-V & 0.52 & 119.2 & 0.5 & $\mathrm{~Wb}$ & VAR \\
\hline 80170 & 5.31 & $\mathrm{~K} 2 \mathrm{III}$ & 1.17 & 0.5 & 0.2 & IAUf & \\
\hline 81797 & 1.99 & K3III & 1.44 & -4.7 & 0.3 & IAUb & \\
\hline 83443 & 8.23 & $\mathrm{~K} 0 \mathrm{~V}$ & 0.81 & 28.7 & 0.3 & $\mathrm{E}$ & \\
\hline 83516 & 8.63 & K0III & 0.97 & 43.5 & 0.2 & $\mathrm{E}$ & \\
\hline 84441 & 2.97 & G0II & 0.81 & 4.5 & 0.4 & IAUb & \\
\hline 86801 & 8.78 & G0V & 0.58 & -11.4 & 1.9 & $\mathrm{H}$ & New SBO \\
\hline 89449 & 4.78 & F6IV & 0.45 & 6.3 & 0.5 & IAUf & VAR \\
\hline 90861 & 6.88 & K2III & 1.11 & 37.1 & 0.3 & $\mathrm{H}$ & \\
\hline 92588 & 6.25 & K1IV & 0.88 & 42.5 & 0.3 & IAUf & \\
\hline 101266 & 9.30 & G5IV & 0.66 & 23.0 & 0.8 & $\mathrm{E}$ & New SBO \\
\hline 102494 & 7.48 & G9IV & 0.87 & -22.1 & 0.3 & $\mathrm{H}$ & \\
\hline 102870 & 3.59 & F8V & 0.52 & 4.3 & 0.3 & IAUb & \\
\hline 103095 & 6.42 & G8V & 0.75 & -98.3 & 0.3 & IAUf & \\
\hline 107328 & 4.97 & K1III & 1.17 & 36.4 & 0.3 & IAUf & \\
\hline 108903 & 1.59 & M4III & 1.60 & 21.0 & 0.4 & $\mathrm{IAUb}$ & IVAR \\
\hline 109379 & 2.65 & G5II & 0.89 & -7.6 & 0.3 & $\mathrm{IAUb}$ & \\
\hline 111417 & 8.30 & K3IV & 1.40 & -19.1 & 0.2 & $\mathrm{E}$ & \\
\hline 112299 & 8.39 & F8V & 0.58 & 3.9 & 0.3 & $\mathrm{H}$ & \\
\hline 114762 & 7.30 & F9V & 0.53 & 49.4 & 0.5 & IAUf & $\mathrm{SBO}$ \\
\hline
\end{tabular}




\begin{tabular}{|c|c|c|c|c|c|c|c|}
\hline HD/DM & $\mathrm{V}$ & $\mathrm{Sp}$ & B-V & $V_{r}$ & $\overline{\sigma_{V r}}$ & list & Rem \\
\hline 115521 & 4.78 & M2III & 1.64 & -28.6 & 2.3 & IAUf & IVAR \\
\hline 120223 & 8.97 & G8IV & 0.97 & -25.1 & 0.5 & $\mathrm{E}$ & Drift \\
\hline 122693 & 8.11 & F8V & 0.59 & -5.5 & 0.3 & $\mathrm{H}$ & \\
\hline 123782 & 5.26 & M2III & 1.64 & -13.9 & 0.6 & IAUf & IVAR \\
\hline 124897 & -0.05 & K2III & 1.24 & -5.3 & 0.3 & IAUb & \\
\hline 126053 & 6.25 & G1V & 0.64 & -19.3 & 0.3 & IAUf & \\
\hline 132737 & 7.64 & K0III & 1.01 & -23.9 & 0.2 & $\mathrm{H}$ & \\
\hline 136202 & 5.04 & F8III-IV & 0.54 & 54.3 & 0.3 & IAUf & \\
\hline 140913 & 8.06 & G0V & 0.61 & -20.0 & 1.1 & $\mathrm{H}$ & SBO \\
\hline 144579 & 6.66 & G8V & 0.73 & -59.5 & 0.3 & IAUf & \\
\hline 145001 & 5.00 & G8III & 0.93 & -10.3 & 0.3 & IAUf & \\
\hline 146051 & 2.73 & M1III & 1.58 & -19.6 & 0.3 & IAUb & \\
\hline 149803 & 8.58 & F7V & 0.48 & -7.5 & 0.7 & $\mathrm{H}$ & VAR \\
\hline 150798 & 1.91 & K2II-III & 1.45 & -3.0 & 0.3 & IAUb & \\
\hline 154417 & 6.00 & F9V & 0.58 & -16.8 & 0.3 & IAUf & \\
\hline 156014 & 2.78 & M5II & 1.16 & -32.0 & 1.2 & IAUb & IVAR \\
\hline 157457 & 5.19 & K1III & 1.05 & 17.8 & 0.3 & IAUf & \\
\hline 160952 & 7.81 & G8III & 0.95 & 26.4 & 1.7 & $\mathrm{H}$ & SBO-IVAR? \\
\hline 161096 & 2.76 & K2III & 1.17 & -12.5 & 0.3 & IAUb & \\
\hline 168454 & 2.72 & K3III & 1.38 & -20.4 & 0.3 & IAUb & \\
\hline 171232 & 7.44 & G8III & 0.88 & -37.3 & 0.8 & $\mathrm{H}$ & Drift \\
\hline 171391 & 5.12 & G8III & 0.93 & 7.4 & 0.2 & IAUf & \\
\hline 176047 & 8.10 & K0III & 0.96 & -42.5 & 0.2 & $\mathrm{E}$ & \\
\hline 182572 & 5.17 & G8IV & 0.76 & -100.4 & 0.3 & IAUf & \\
\hline 184467 & 6.60 & $\mathrm{~K} 1 \mathrm{~V}$ & 0.86 & 11.2 & 9.9 & IAUf & SB2O \\
\hline 184499 & 6.62 & G0V & 0.59 & -166.1 & 0.5 & $\mathrm{~Wb}$ & VAR? SB? \\
\hline 186791 & 2.72 & K3II & 1.51 & -2.8 & 0.6 & IAUb & IVAR \\
\hline 187691 & 5.12 & F8V & 0.56 & -0.0 & 0.3 & IAUf & \\
\hline 193231 & 8.39 & G5V... & 0.73 & -31.8 & 1.5 & $\mathrm{E}$ & Drift \\
\hline 194071 & 7.9 & G8III & 0.60 & -9.4 & 0.3 & $\mathrm{H}$ & \\
\hline 194598 & 8.33 & F7V-VI & 0.50 & -246.5 & 0.9 & $\mathrm{~Wb}$ & VAR \\
\hline 196983 & 9.08 & K2III & 1.18 & -9.1 & 0.3 & $\mathrm{E}$ & \\
\hline 203638 & 5.38 & K0III & 1.18 & 22.1 & 0.2 & IAUf & \\
\hline 204867 & 2.90 & G0Ib & 0.83 & 6.3 & 0.3 & IAUb & \\
\hline 204934 & 8.20 & K1III & 1.10 & -4.8 & 4.9 & $\mathrm{H}$ & SBO-IVAR? \\
\hline 206778 & 2.38 & $\mathrm{~K} 2 \mathrm{Ib}$ & 1.52 & 3.4 & 0.6 & IAUb & IVAR \\
\hline 212943 & 4.78 & K0III & 1.04 & 54.2 & 0.2 & IAUf & \\
\hline 213014 & 7.45 & G9III & 0.89 & -39.9 & 0.2 & IAUf & \\
\hline 213947 & 6.88 & K2 & 1.48 & 16.5 & 0.3 & $\mathrm{H}$ & \\
\hline 219509 & 8.71 & K5 & 1.05 & 67.5 & 0.5 & $\mathrm{E}$ & Drift \\
\hline 222368 & 4.13 & F7V & 0.51 & 5.6 & 0.3 & IAUb & \\
\hline 223094 & 6.97 & K5III & 1.63 & 20.2 & 0.4 & $\mathrm{H}$ & IVAR \\
\hline 223311 & 6.09 & K4III & 1.45 & -20.2 & 0.3 & IAUf & \\
\hline 223647 & 5.10 & G7III & 0.93 & 15.4 & 0.6 & IAUf & Drift \\
\hline
\end{tabular}




\section{Discussion}

Walker: With all the considerable efforts now being devoted to radial-velocity standards it is really important to monitor, simultaneously, chromospheric activity. Otherwise the question of intrinsic versus binary variability will keep coming up.

Udry: We are planning to do so with ELODIE.

Isaak: Adding to Gordon Walker's comment on monitoring chromospheric activity, one should monitor the velocity frequently in order comfortably to satisfy the Nyquist criterion for the activity-induced velocity modulation we discovered on the sun in 1982 . 